DOI: http://dx.doi.org/10.18273/revint.v36n2-2018006

\title{
Multilinear analysis for discrete and periodic pseudo-differential operators in $L^{p}$-spaces
}

\author{
Duván CARdona $^{a *}$, Vishvesh Kumar ${ }^{b}$ \\ ${ }^{a}$ Pontificia Universidad Javeriana, Department of Mathematics, Bogotá, Colombia. \\ ${ }^{b}$ Indian Institute of Technology Delhi, Department of Mathematics, New \\ Delhi-110016, India.
}

\begin{abstract}
In this note we announce our investigation on the $L^{p}$ properties for periodic and discrete multilinear pseudo-differential operators. First, we review the periodic analysis of multilinear pseudo-differential operators by showing classical multilinear Fourier multipliers theorems (proved by Coifman and Meyer, Tomita, Miyachi, Fujita, Grafakos, Tao, etc.) in the context of periodic and discrete multilinear pseudo-differential operators. For this, we use the periodic analysis of pseudo-differential operators developed by Ruzhansky and Turunen. The $s$-nuclearity, $0<s \leq 1$, for the discrete and periodic multilinear pseudo-differential operators will be investigated. To do so, we classify those $s$-nuclear, $0<s \leq 1$, multilinear integral operators on arbitrary Lebesgue spaces defined on $\sigma$-finite measures spaces. Finally, we present some applications of our analysis to deduce the periodic Kato-Ponce inequality and to examine the $s$-nuclearity of multilinear Bessel potentials as well as the $s$-nuclearity of periodic Fourier integral operators admitting suitable types of singularities.
\end{abstract}

Keywords: Pseudo-differential operator, discrete operator, periodic operator, nuclearity, boundedness, Fourier integral operator, multilinear analysis.

MSC2010: 58J40, 47B10, 47G30, 35S30.

\section{Análisis multilineal para operadores pseudodiferenciales periódicos y discretos en espacios $L^{p}$}

Resumen. En esta nota anunciamos los resultados de nuestra investigación sobre las propiedades $L^{p}$ de operadores pseudodiferenciales multilineales periódicos y/o discretos. Primero, revisaremos el análisis multilineal de tales operadores mostrando versiones análogas de los teoremas clásicos disponibles en el análisis multilineal euclidiano (debidos a Coifman y Meyer, Tomita,

*E-mail: duvanc306@gmail.com

Received: 24 September 2018, Accepted: 29 November 2018.

To cite this article: D. Cardona, V. Kumar, Multilinear analysis for discrete and periodic pseudo-differential operators in $L^{p}$-spaces, Rev. Integr. temas mat. 36 (2018), No. 2, 151-164. doi: 10.18273/revint.v36n22018006. 
Miyachi, Fujita, Grafakos, Tao, etc.), pero, en el contexto de operadores periódicos y/o discretos. Se caracterizará la $s$-nuclearidad, $0<s \leq 1$, para operadores multilineales pseudodiferenciales periódicos y/o discretos. Para cumplir este objetivo se clasificarán aquellos operadores lineales $s$-nucleares, $0<s \leq 1$, multilineales con núcleo, sobre espacios de Lebesgue arbitrarios definidos en espacios de medida $\sigma$-finitos. Finalmente, como aplicación de los resultados presentados se obtiene la versión periódica de la desigualdad de Kato-Ponce, y se examina la $s$-nuclearidad de potenciales de Bessel lineales y multilineales, como también la $s$-nuclearidad de operadores integrales de Fourier periódicos admitiendo símbolos con tipos adecuados de singularidad. Palabras clave: Operador pseudo-diferencial, operador discreto, operador periódico, nuclearidad, continuidad, operador integral de Fourier, Análisis multilineal.

\section{Introduction}

The goal of this note is to announce the main results about the $L^{p}$-multilinear analysis developed by the authors in [10] for periodic and discrete pseudo-differential operators. These operators can be defined by using the multilinear Fourier transform as follows. If $m: \mathbb{T}^{n} \times \mathbb{Z}^{n r} \rightarrow \mathbb{C}, \mathbb{T}^{n} \cong[0,1)^{n}$ is a suitable function, then the periodic multilinearpseudo-differential operator associated to $m$ is the operator defined as

$$
T_{m}(f)(x)=\sum_{\xi \in \mathbb{Z}^{n r}} e^{i 2 \pi x \cdot\left(\xi_{1}+\xi_{2}+\cdots+\xi_{r}\right)} m(x, \xi)\left(\mathscr{F}_{\mathbb{T}^{n}} f_{1}\right)\left(\xi_{1}\right) \cdots\left(\mathscr{F}_{\mathbb{T}^{n}} f_{r}\right)\left(\xi_{r}\right), x \in \mathbb{T}^{n}
$$

where $f=\left(f_{1}, \cdots, f_{r}\right) \in \mathscr{D}\left(\mathbb{T}^{n}\right)^{r}$, and

$$
(\mathscr{F} f)(\xi):=\prod_{j=1}^{r}\left(\mathscr{F}_{\mathbb{T}^{n}} f_{j}\right)\left(\xi_{j}\right)=\prod_{j=1}^{r} \int_{\mathbb{T}^{n}} e^{-i 2 \pi x_{j} \xi_{j}} f_{j}\left(x_{j}\right) d x_{j}, \xi=\left(\xi_{1}, \cdots, \xi_{r}\right) \in \mathbb{Z}^{n r}
$$

is the periodic multilinear Fourier transform of $f$. We have denoted by $\mathscr{D}\left(\mathbb{T}^{n}\right)$ the space of smooth functions on the torus $C^{\infty}\left(\mathbb{T}^{n}\right)$ endowed with its usual Fréchet structure. On the other hand, if $a: \mathbb{Z}^{n} \times \mathbb{T}^{n r} \rightarrow \mathbb{C}$ is a measurable function, then the discrete multilinearpseudo-differential operator associated to $a$ is the multilinear operator defined by

$$
T_{a}(g)(\ell)=\int_{\mathbb{T}^{n r}} e^{i 2 \pi \ell \cdot\left(\eta_{1}+\cdots+\eta_{r}\right)} a(\ell, \eta)\left(\mathscr{F}_{\mathbb{Z}^{n}} g_{1}\right)\left(\eta_{1}\right) \cdots\left(\mathscr{F}_{\mathbb{Z}^{n}} g_{r}\right)\left(\eta_{r}\right) d \eta, \quad \ell \in \mathbb{Z}^{n}
$$

where $g=\left(g_{1}, \cdots, g_{r}\right) \in \mathscr{S}\left(\mathbb{Z}^{n}\right)^{r}$, and $\left(\mathscr{F}_{\mathbb{Z}^{n}} g_{i}\right)\left(\eta_{i}\right)=\sum_{\ell_{i} \in \mathbb{Z}^{n}} e^{-i 2 \pi \ell_{i} \eta_{i}} g_{i}\left(\ell_{i}\right), \eta_{i} \in \mathbb{T}^{n}$ is the discrete Fourier transform of $g_{i}$. For $r \geq 2$, these operators have been studied by V. Catan $\breve{a}$ in [12]. If $r=1$, these quantization formulae can be reduced to the known expressions

$$
T_{m}(f)(x)=\sum_{\xi \in \mathbb{Z}^{n}} e^{i 2 \pi x \cdot \xi} m(x, \xi)\left(\mathscr{F}_{\mathbb{T}^{n}} f\right)(\xi), x \in \mathbb{T}^{n}
$$

[Revista Integración, temas de matemáticas 
and

$$
T_{a}(g)(\ell)=\int_{\mathbb{T}^{n}} e^{i 2 \pi \ell \cdot \eta} a(\ell, \eta)\left(\mathscr{F}_{\mathbb{Z}^{n}} g\right)(\eta) d \eta, \quad \ell \in \mathbb{Z}^{n}
$$

Periodic pseudo-differential operators (see (3)) were defined by Volevich and Agranovich [1]. The subsequent works of McLean [29], Turunen and Vainikko [47], and Ruzhansky and Turunen [44] developed a periodic analysis from Hörmander classes to applications to periodic equations, although the symbolic calculus was consistently developed by Ruzhansky and Turunen [44]. Nevertheless, the references Ruzhansky and Turunen [44], [45], Cardona [6], [7], [8], Delgado [15] and Molahajloo and Wong [34], [35], [36] provide some complementary results for the subject. Mapping properties for more general operators as periodic Fourier integral operators appear in Ruzhansky and Turunen [45] and Cardona, Messiouene and Senoussaoui [11].

In a more recent approach, pseudo-differential operators on $\mathbb{Z}^{n}$ (discrete pseudodifferential operators) were introduced by Molahajloo in [36], and some of its properties were developed in the last years in the references [9], [17], [28], [38], [39], [40], [41], [43]. However, only the fundamental work L. Botchway, G. Kibiti, and M. Ruzhansky [5] includes properties about a discrete pseudo-differential calculus and applications to difference equations. The reference [9] discusses those relations of the theory of discrete pseudo-differential operators with important problems in number theory as the Waring problem and the hypothesis $K^{*}$ by Hooley.

An overview to the mapping properties for pseudo-differential operators on $\mathbb{R}^{n}$ provides the expected results in the discrete and periodic setting. On $\mathbb{R}^{n}$ these operators have the form

$$
A f(x)=\int_{\mathbb{R}^{n}} e^{i 2 \pi x \cdot \xi} a(x, \xi) \widehat{f}(\xi) d \xi, \quad f \in \mathscr{D}\left(\mathbb{R}^{n}\right),
$$

with $\widehat{f}$ the euclidean Fourier transform of $f$ (see Hörmander [25]). The nuclearity of pseudo-differential operators on $\mathbb{R}^{n}$ has been treated in Aoki and Rempala [2], [42]. In a context closely related to our work, multilinear pseudo-differential operators have been treated in Bényi, Maldonado, Naibo, and Torres, [3], [4], Michalowski, Rule and Staubach, Miyachi and Tomita [30], [31], [32], [33] and references therein. The multilinear analysis for multilinear Fourier multipliers

$$
T_{a}(f)(x)=\int_{\mathbb{R}^{n r}} e^{i 2 \pi x \cdot\left(\eta_{1}+\cdots+\eta_{r}\right)} a(\eta) \widehat{f}_{1}\left(\eta_{1}\right) \cdots \widehat{f}_{r}\left(\eta_{r}\right)\left(\eta_{r}\right) d \eta, x \in \mathbb{R}^{n}
$$

born with the works of Coifman and Meyer [13], [14], where the condition

$$
\left|\partial_{\eta_{1}}^{\alpha_{1}} \partial_{\eta_{2}}^{\alpha_{2}} \cdots \partial_{\eta_{r}}^{\alpha_{r}} a\left(\eta_{1}, \eta_{2}, \cdots, \eta_{r}\right)\right| \leq C_{\alpha}\left(\left|\eta_{1}\right|+\left|\eta_{2}\right|+\cdots+\left|\eta_{r}\right|\right)^{-|\alpha|},
$$

for sufficiently many multi-indices $\alpha=\left(\alpha_{1}, \alpha_{2}, \cdots, \alpha_{r}\right)$, was proved to be sufficient for the boundedness of $T_{a}$ from $L^{p_{1}}\left(\mathbb{R}^{n}\right) \times L^{p_{2}}\left(\mathbb{R}^{n}\right) \times \cdots \times L^{p_{r}}\left(\mathbb{R}^{n}\right)$ into $L^{p}\left(\mathbb{R}^{n}\right)$ provided that $1 / p=1 / p_{1}+1 / p_{2}+\cdots+1 / p_{r}$, and $1 \leq p_{i}, p<\infty$. A generalization for this result was obtained by Tomita in [46], where it was proved that the multilinear Hörmander condition

$$
\|a\|_{l . u ., H_{l o c}^{s}\left(\mathbb{R}^{n r}\right)}:=\sup _{k \in \mathbb{Z}}\left\|a\left(2^{k} \eta_{1}, 2^{k} \eta_{2}, \cdots, 2^{k} \eta_{r}\right) \phi\right\|_{H^{s}}<\infty, \phi \in \mathscr{D}(0, \infty), s>\frac{n r}{2},
$$

Vol. 36, $\left.\mathrm{N}^{\circ} 2,2018\right]$ 
implies the boundedness of $T_{a}$ from $L^{p_{1}}\left(\mathbb{R}^{n}\right) \times L^{p_{2}}\left(\mathbb{R}^{n}\right) \times \cdots \times L^{p_{r}}\left(\mathbb{R}^{n}\right)$ into $L^{p}\left(\mathbb{R}^{n}\right)$ provided that $1 / p=1 / p_{1}+1 / p_{2}+\cdots+1 / p_{r}$, and $1 \leq p_{i}, p<\infty$. The case $r=1$ is known as the Hörmander-Mihlin theorem. These multilinear theorems have been generalized to Hardy spaces $H^{p}\left(\mathbb{R}^{n}\right)$ for suitable values of $0<p_{i}, p<\infty$, in the works of Grafakos, Torres, Miyachi, Fujita, Tomita, Kenig, Stein, Muscalo, Thiele and Tao [19], [21], [22], [23], [24], [27], [37]. The main novelty of this work is that we provide discrete and periodic analogues for these works in the multilinear setting.

This note is organized as follows. In section 2 we provide those results on the boundedness of pseudo-differential operators on $\mathbb{Z}^{n}$ and the torus. Later, in Section 3 we classify those $s$-nuclear multilinear integral operators on arbitrary $\sigma$-finite measure spaces and we apply this classification to periodic and discrete multilinear pseudo-differential operators.

\section{Boundedness of pseudo-differential operators on $\mathbb{T}^{n}$ and $\mathbb{Z}^{n}$}

In this section we explain in detail the main results of our investigation on the boundedness of the multilinear operators considered. Our starting point is the following multilinear version of the Stein-Weiss multiplier theorem (see Theorem 3.8 of Stein and Weiss [48]). Sometimes we denote $(x, \xi):=\left(x, \xi_{1}, \cdots, \xi_{r}\right)=x \cdot\left(\xi_{1}+\cdots+\xi_{r}\right)$.

Theorem 2.1. Let $1<p<\infty$ and let $a: \mathbb{R}^{n r} \rightarrow \mathbb{C}$ be a continuous bounded function. If the multilinear Fourier multiplier operator

$$
T f(x)=\int_{\mathbb{R}^{n r}} e^{i 2 \pi\left(x, \xi_{1}, \xi_{2}, \cdots, \xi_{r}\right)} a\left(\xi_{1}, \xi_{2}, \cdots \xi_{r}\right) \widehat{f}_{1}\left(\xi_{1}\right) \cdots \widehat{f}_{r}\left(\xi_{r}\right) d \xi
$$

extends to a bounded multilinear operator from $L^{p_{1}}\left(\mathbb{R}^{n}\right) \times L^{p_{2}}\left(\mathbb{R}^{n}\right) \times \cdots \times L^{p_{r}}\left(\mathbb{R}^{n}\right)$ into $L^{p}\left(\mathbb{R}^{n}\right)$, then the periodic multilinear Fourier multiplier

$$
A f(x):=\sum_{\xi \in \mathbb{Z}^{n r}} e^{i 2 \pi\left(x, \xi_{1}, \xi_{2}, \cdots, \xi_{r}\right)} a\left(\xi_{1}, \xi_{2}, \cdots, \xi_{r}\right)\left(\mathscr{F}_{\mathbb{T}^{n}} f_{1}\right)\left(\xi_{1}\right) \cdots\left(\mathscr{F}_{\mathbb{T}^{n}} f_{r}\right)\left(\xi_{r}\right)
$$

also extends to a bounded multilinear operator from $L^{p_{1}}\left(\mathbb{T}^{n}\right) \times L^{p_{2}}\left(\mathbb{T}^{n}\right) \times \cdots \times L^{p_{r}}\left(\mathbb{T}^{n}\right)$ into $L^{p}\left(\mathbb{T}^{n}\right)$, provided that

$$
\frac{1}{p_{1}}+\cdots+\frac{1}{p_{r}}=\frac{1}{p}, 1 \leq p_{i}<\infty
$$

Moreover, there exists a positive constant $C_{p}$ such that the following inequality holds:

$$
\|A\|_{\mathscr{B}\left(L^{p_{1}}\left(\mathbb{T}^{n}\right) \times L^{p_{2}}\left(\mathbb{T}^{n}\right) \times \cdots \times L^{p_{r}}\left(\mathbb{T}^{n}\right), L^{p}\left(\mathbb{T}^{n}\right)\right)} \leq C_{p}\|T\|_{\mathscr{B}\left(L^{p_{1}}\left(\mathbb{R}^{n}\right) \times L^{p_{2}}\left(\mathbb{R}^{n}\right) \times \cdots \times L^{p_{r}}\left(\mathbb{R}^{n}\right), L^{p}\left(\mathbb{R}^{n}\right)\right)} .
$$

Remark 2.2. Theorem 2.1 can be proved in the following way. By the density of the trigonometric polynomials, we can prove that under the conditions of this theorem, we have the estimate

$$
\left\|A\left(P_{1}, P_{2}, \cdots, P_{r}\right)\right\|_{\left.L^{p}\left(\mathbb{T}^{n}\right)\right)} \leq C \prod_{j=1}^{r}\left\|P_{j}\right\|_{L^{p_{j}}\left(\mathbb{T}^{n}\right)},
$$


where the constant $C$ does not dependent of every trigonometric polynomial $P_{i}$. For this, we will prove that

$$
\begin{aligned}
\lim _{\varepsilon \rightarrow 0} \varepsilon^{n / 2} \int_{\mathbb{R}^{n}} & \left(T\left(P_{1} w_{\alpha_{1} \varepsilon}, P_{2} w_{\alpha_{2} \varepsilon}, \cdots, P_{r} w_{\alpha_{r} \varepsilon}\right)\right)(x) \overline{Q(x)} w_{\varepsilon \beta}(x) d x \\
= & c_{n, r, p} \int_{\mathbb{T}^{n}} A\left(P_{1}, P_{2}, \cdots, P_{r}\right) \overline{Q(x)} d x, \quad w_{\delta}(x)=e^{-\delta|x|^{2}}, \quad \delta>0,
\end{aligned}
$$

for some positive constant $c_{n, r, p}>0$. We will assume that

$$
\sum_{j=1}^{r} \alpha_{j}+\beta=1, \quad \alpha_{i}, \beta>0 .
$$

Observe that by linearity, we only need to prove (10) when $P_{i}\left(x_{i}\right)=e^{i 2 \pi m_{i} x_{i}}$ and $Q(x)=$ $e^{i 2 \pi k x}$ for $k$ and $m_{i}$ in $\mathbb{Z}^{n}, 1 \leq i \leq r$. The main step in our proof (see Cardona and Kumar [10]) is to show (10) and how it implies (9).

With the help of the previous result we prove the following fact. We use the notation

$$
\langle\xi\rangle:=\max \left\{1,\left|\xi_{1}\right|+\cdots+\left|\xi_{r}\right|\right\},
$$

for all $\xi \in \mathbb{R}^{n r}$. Now, we provide the following discrete version of the known result of Coifman and Meyer mentioned in the introduction.

Theorem 2.3. Let $T_{m}$ be a periodic multilinear Fourier multiplier. Under the condition

$$
\left|\Delta_{\xi_{1}}^{\alpha_{1}} \cdots \Delta_{\xi_{r}}^{\alpha_{r}} m\left(\xi_{1}, \xi_{2}, \cdots, \xi_{r}\right)\right| \leq C_{\alpha}\langle\xi\rangle^{-\left|\alpha_{1}\right|-\cdots-\left|\alpha_{r}\right|}, \quad|\alpha| \leq\left[\frac{n r}{2}\right]+1
$$

the operator $T_{m}$ extends to a bounded multilinear operator from $L^{p_{1}}\left(\mathbb{T}^{n}\right) \times L^{p_{2}}\left(\mathbb{T}^{n}\right) \times$ $\cdots \times L^{p_{r}}\left(\mathbb{T}^{n}\right)$ into $L^{p}\left(\mathbb{T}^{n}\right)$, provided that

$$
\frac{1}{p_{1}}+\cdots+\frac{1}{p_{r}}=\frac{1}{p}, 1 \leq p_{i}<\infty .
$$

If we consider Fourier integral operators (FIOs) with periodic phases, we can recover the following multilinear version for FIOs of the multiplier theorem of Stein and Weiss.

Theorem 2.4. Let $1<p<\infty$ and let $\phi$ be a real valued continuous function defined on $\mathbb{T}^{n} \times \mathbb{R}^{n r}$. If a : $\mathbb{T}^{n} \times \mathbb{R}^{n r} \rightarrow \mathbb{C}$ is a continuous bounded function, and the multilinear Fourier integral operator

$$
T f(x)=\int_{\mathbb{R}^{n r}} e^{i \phi\left(x, \xi_{1}, \xi_{2}, \cdots, \xi_{r}\right)} a\left(x, \xi_{1}, \xi_{2}, \cdots, \xi_{r}\right) \widehat{f}_{1}\left(\xi_{1}\right) \cdots \widehat{f}_{r}\left(\xi_{r}\right) d \xi
$$

extends to a bounded multilinear operator from $L^{p_{1}}\left(\mathbb{R}^{n}\right) \times L^{p_{2}}\left(\mathbb{R}^{n}\right) \times \cdots \times L^{p_{r}}\left(\mathbb{R}^{n}\right)$ into $L^{p}\left(\mathbb{R}^{n}\right)$, then the periodic multilinear Fourier integral operator

$$
A f(x):=\sum_{\xi \in \mathbb{Z}^{n r}} e^{i \phi\left(x, \xi_{1}, \xi_{2}, \cdots, \xi_{r}\right)} a\left(x, \xi_{1}, \xi_{2}, \cdots, \xi_{r}\right)\left(\mathscr{F}_{\mathbb{T}^{n}} f_{1}\right)\left(\xi_{1}\right) \cdots\left(\mathscr{F}_{\mathbb{T}^{n}} f_{r}\right)\left(\xi_{r}\right)
$$

Vol. 36, $\left.\mathrm{N}^{\circ} 2,2018\right]$ 
also extends to a bounded multilinear operator from $L^{p_{1}}\left(\mathbb{T}^{n}\right) \times L^{p_{2}}\left(\mathbb{T}^{n}\right) \times \cdots \times L^{p_{r}}\left(\mathbb{T}^{n}\right)$ into $L^{p}\left(\mathbb{T}^{n}\right)$, provided that

$$
\frac{1}{p_{1}}+\cdots+\frac{1}{p_{r}}=\frac{1}{p}, 1 \leq p_{i}<\infty .
$$

Moreover, there exists a positive constant $C_{p}$ such that

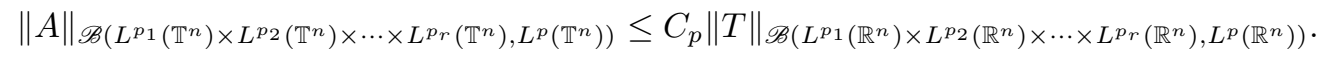

Now, we present some results about the boundedness of periodic multilinear pseudodifferential operators where explicit conditions on the multilinear symbols are considered.

Theorem 2.5. Let us assume that $m$ satisfies the Hörmander condition of order $s>0$,

$$
\|m\|_{L^{\infty}\left(\mathbb{T}^{n}, l . u ., H_{l o c}^{s}\left(\mathbb{R}^{n}\right)\right)}:=\operatorname{ess} \sup _{x \in \mathbb{T}^{n}}\|m(x, \cdot)\|_{l . u ., H_{l o c}^{s}}<\infty .
$$

Then the multilinear periodic pseudo-differential operator $T_{m}$ associated with $m$ extends to a bounded operator from $L^{p_{1}}\left(\mathbb{T}^{n}\right) \times L^{p_{2}}\left(\mathbb{T}^{n}\right) \times \cdots \times L^{p_{r}}\left(\mathbb{T}^{n}\right)$ into $L^{p}\left(\mathbb{T}^{n}\right)$ provided that $s>\frac{3 n r}{2}$ and

$$
\frac{1}{p}=\frac{1}{p_{1}}+\cdots+\frac{1}{p_{r}}, 1 \leq p<\infty, 1 \leq p_{i} \leq \infty .
$$

Remark 2.6. The proof of Theorem 2.5 is based on a suitable Littlewood-Paley decomposition of the symbol $m$. Indeed, we decompose $m$ as

$$
m=\sum_{j=1}^{\infty} m_{j}, \operatorname{supp}\left(m_{j}\right) \subset\left[2^{j}, 2^{j+1}\right] .
$$

We prove that by assuming (11), we can decompose the operator $T_{m}$ as

$$
T_{m} f=\sum_{j=1}^{\infty} T_{m_{j}} f, f=\left(f_{1}, \cdots, f_{r}\right) \in \mathscr{D}\left(\mathbb{T}^{n}\right)^{r}
$$

where every operator $T_{m_{j}}$ is associated to the symbol $m_{j}$, and we prove that the operator norm of every $T_{m_{j}}$ is less than $\|m\|_{L^{\infty}\left(\mathbb{T}^{n}, l . u ., H_{\text {loc }}^{s}\left(\mathbb{R}^{n}\right)\right)}$ multiplied by a factor proportional to $2^{-j\left(s-\frac{3 n}{2}\right)}$. We conclude our proof in [10] by observing that $\|T\|_{\mathscr{B}\left(L^{p_{1}} \times L^{p_{2}} \times \cdots \times L^{p_{r}}, L^{p}\right)} \leq$ $\sum_{j}\left\|T_{m_{j}}\right\|_{\mathscr{B}\left(L^{p_{1}} \times L^{p_{2}} \times \cdots \times L^{p_{r}}, L^{p}\right)}$.

The following theorem is an extension of the Coifman-Meyer result presented above in the multilinear pseudo-differential framework.

Theorem 2.7. Let us assume that $m$ satisfies the discrete symbol inequalities

$$
\sup _{x \in \mathbb{T}^{n}}\left|\Delta_{\xi_{1}}^{\alpha_{1}} \Delta_{\xi_{2}}^{\alpha_{2}} \cdots \Delta_{\xi_{r}}^{\alpha_{r}} m\left(x, \xi_{1}, \cdots, \xi_{r}\right)\right| \leq C_{\alpha}\langle\xi\rangle^{-|\alpha|},
$$

for all $|\alpha|:=\left|\alpha_{1}\right|+\cdots+\cdots+\left|\alpha_{r}\right| \leq[3 n r / 2]+1$. Then the periodic multilinear pseudodifferential operator $T_{m}$ extends to a bounded operator from $L^{p_{1}}\left(\mathbb{T}^{n}\right) \times L^{p_{2}}\left(\mathbb{T}^{n}\right) \times \cdots \times$ $L^{p_{r}}\left(\mathbb{T}^{n}\right)$ into $L^{p}\left(\mathbb{T}^{n}\right)$, provided that

$$
\frac{1}{p}=\frac{1}{p_{1}}+\cdots+\frac{1}{p_{r}}, 1 \leq p<\infty, 1 \leq p_{i} \leq \infty .
$$

[Revista Integración, temas de matemáticas 
Remark 2.8. We prove Theorem 2.7 by observing that (14) implies (11). We develop this delicate argument in [10] where we use, among other things, the periodic analysis developed by Ruzhansky and Turunen.

The condition on the number of discrete derivatives in the preceding result can be relaxed if we assume regularity in $x$. We show it in the following theorem.

Theorem 2.9. Let $T_{m}$ be a periodic multilinear pseudo-differential operator. If $m$ satisfies toroidal conditions of the type

$$
\left|\partial_{x}^{\beta} \Delta_{\xi_{1}}^{\alpha_{1}} \cdots \Delta_{\xi_{r}}^{\alpha_{r}} m\left(x, \xi_{1}, \xi_{2}, \cdots, \xi_{r}\right)\right| \leq C_{\alpha}\langle\xi\rangle^{-\left|\alpha_{1}\right|-\cdots-\left|\alpha_{r}\right|},
$$

where $|\alpha| \leq\left[\frac{n r}{2}\right]+1$, and $|\beta| \leq\left[\frac{n}{p}\right]+1$, then $T_{m}$ extends to a bounded multilinear operator from $L^{p_{1}}\left(\mathbb{T}^{n}\right) \times L^{p_{2}}\left(\mathbb{T}^{n}\right) \times \cdots \times L^{p_{r}}\left(\mathbb{T}^{n}\right)$ into $L^{p}\left(\mathbb{T}^{n}\right)$, provided that

$$
\frac{1}{p_{1}}+\cdots+\frac{1}{p_{r}}=\frac{1}{p}, 1 \leq p_{i}<\infty .
$$

Example 2.10. Theorem 2.7 applied to the bilinear operator

$$
B_{s}(f, g):=J^{s}(f \cdot g),
$$

where $J^{s}$ is the periodic fractional derivative operator $(\mathcal{L})^{s / 2}$, or the periodic Bessel potential of order $s>0,(1+\mathcal{L})^{s / 2}$, implies the (well known) periodic Kato-Ponce inequality:

$$
\left\|J^{s}(f \cdot g)\right\|_{L^{r}\left(\mathbb{T}^{n}\right)} \lesssim\left\|J^{s} f\right\|_{L^{p_{1}\left(\mathbb{T}^{n}\right)}}\|g\|_{L^{q_{1}\left(\mathbb{T}^{n}\right)}}+\|f\|_{L^{p_{2}\left(\mathbb{T}^{n}\right)}}\left\|J^{s} g\right\|_{L^{q_{2}\left(\mathbb{T}^{n}\right)}},
$$

where $\frac{1}{p_{1}}+\frac{1}{q_{1}}=\frac{1}{p_{2}}+\frac{1}{q_{2}}=\frac{1}{r}, 1<r<\infty, 1 \leq p_{i}, q_{i} \leq \infty$, and $\mathcal{L}=-\frac{1}{4 \pi^{2}}\left(\sum_{j=1}^{n} \partial_{\theta_{j}}^{2}\right)$ is the Laplacian on the torus.

Boundedness of discrete multilinear pseudo-differential operators. Our main results about the boundedness of discrete multilinear pseudo-differential operators are stated as follows.

Theorem 2.11. Let $\sigma \in L^{\infty}\left(\mathbb{Z}^{n}, C^{2 \varkappa}\left(\mathbb{T}^{n r}\right)\right)$. If $\sigma$ satisfies the discrete inequality

$$
\left|\partial_{\xi}^{\beta} \sigma(\ell, \xi)\right| \leq C_{\beta}, \ell \in \mathbb{Z}^{n}, \xi \in \mathbb{T}^{n r}, \sigma(\ell, \xi)=\sigma(\ell)(\xi),
$$

for all $\beta$ with $|\beta|=2 \varkappa$, then $T_{\sigma}$ extends to a bounded operator from $L^{p_{1}}\left(\mathbb{Z}^{n}\right) \times L^{p_{2}}\left(\mathbb{Z}^{n}\right) \times$ $\cdots \times L^{p_{r}}\left(\mathbb{Z}^{n}\right)$ into $L^{s}\left(\mathbb{Z}^{n}\right)$, provided that $1 \leq p_{j} \leq p \leq \infty$, and

$$
\frac{1}{s}-\frac{1}{p}<\frac{2 \varkappa}{n r}-1
$$

The following result can be derived of the previous result with $r=1$ and $s=p$.

Corollary 2.12. Let $\sigma \in C^{2 \varkappa}\left(\mathbb{Z}^{n} \times \mathbb{T}^{n}\right)$. If $\sigma$ satisfies the discrete inequality

$$
\left|\partial_{\xi}^{\beta} \sigma(\ell, \xi)\right| \leq C_{\beta}, \ell \in \mathbb{Z}^{n}, \xi \in \mathbb{T}^{n},
$$

for all $\beta$ with $|\beta|=2 \varkappa$, then $T_{\sigma}$ extends to a bounded operator from $L^{p}\left(\mathbb{Z}^{n}\right)$ into $L^{p}\left(\mathbb{Z}^{n}\right)$, provided that $1 \leq p \leq \infty$, and $\varkappa>n / 2$.

Vol. 36, $\left.\mathrm{N}^{\circ} 2,2018\right]$ 


\section{3. $s$-Nuclearity, $0<s \leq 1$, of pseudo-differential operators on $\mathbb{T}^{n}$ and $\mathbb{Z}^{n}$}

In this section we study the $s$-nuclearity, $0<s \leq 1$ of multilinear discrete and periodic pseudo-differential operators. We prove Theorem 3.1 regarding the characterization of $s$-nuclear multilinear operators on abstract $\sigma$-finite measure spaces, and Theorem 3.2 and Theorem 3.3 regarding the characterization of $s$-nuclearity of periodic and discrete pseudo-differential operators. Although these theorems are multilinear extensions of the results due to Delgado [16], Delgado and Wong [17], JamalpourBirgani [26] and Ghaemi, JamalpourBirgani and Wong [20], we can recover their results from our results by considering $r=1$. In order to study these multilinear operators admitting $s$-nuclear extensions, we prove the following multilinear version of a result by Delgado, on the nuclearity of integral operators on Lebesgue spaces (see [16], [18]). So, in the following multilinear theorem we characterize those $s$-nuclear (multilinear) integral operators on arbitrary $(\sigma$-finite) measure spaces $(X, \mu)$.

Theorem 3.1. Let $\left(X_{i}, \mu_{i}\right), 1 \leq i \leq r$ and $(Y, \nu)$ be $\sigma$-finite measure spaces. Let $1 \leq$ $p_{i}, p<\infty, 1 \leq i \leq r$ and let $p_{i}^{\prime}, q$ be such that $\frac{1}{p_{i}}+\frac{1}{p_{i}^{\prime}}=1, \frac{1}{p}+\frac{1}{q}=1$ for $1 \leq i \leq r$. Let $T: L^{p_{1}}(\mu) \times L^{p_{2}}\left(\mu_{2}\right) \times \cdots \times L^{p_{r}}\left(\mu_{r}\right) \rightarrow L^{p}(\nu)$ be a multilinear operator. Then $T$ is a $s$-nuclear, $0<s \leq 1$, operator if, and only if, there exist sequences $\left\{g_{n}\right\}_{n}$ with $g_{n}=\left(g_{n 1}, g_{n 2}, \ldots, g_{n r}\right)$ and $\left\{h_{n}\right\}_{n}$ in $L^{p_{1}^{\prime}}\left(\mu_{1}\right) \times L^{p_{2}^{\prime}}\left(\mu_{2}\right) \times \cdots \times L^{p_{r}^{\prime}}\left(\mu_{r}\right)$ and $L^{p}(\nu)$,

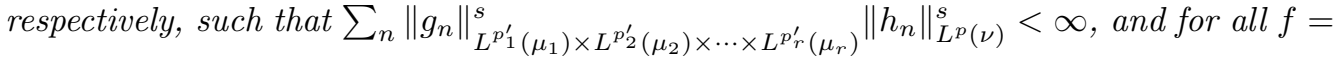
$\left(f_{1}, f_{2}, \ldots, f_{r}\right) \in L^{p_{1}}(\mu) \times L^{p_{2}}\left(\mu_{2}\right) \times \cdots \times L^{p_{r}}\left(\mu_{r}\right)$ we have

$$
\begin{aligned}
(T f)(y)= & \int_{X_{1}, X_{2}, \cdots, X_{r}}\left(\sum_{n=1}^{\infty} g_{n}(x) h_{n}(y)\right) f(x) d\left(\mu_{1} \otimes \mu_{2} \otimes \cdots \otimes \mu_{r}\right)(x) \\
= & \int_{X_{1}} \int_{X_{2}} \cdots \int_{X_{r}}\left(\sum_{n=1}^{\infty} g_{n 1}\left(x_{1}\right) g_{n 2}\left(x_{2}\right) \ldots g_{n r}\left(x_{r}\right) h_{n}(y)\right) \\
& \times f_{1}\left(x_{1}\right) f_{2}\left(x_{2}\right) \ldots f_{r}\left(x_{r}\right) d \mu_{1}\left(x_{1}\right) d \mu_{2}\left(x_{2}\right) \cdots d \mu_{r}\left(x_{r}\right)
\end{aligned}
$$

for almost every $y \in Y$.

Remark 3.2. The proof of Theorem 3.1 is based on an important lemma proved in $[10$, Lemma 4.1]. The proof of the if part of Theorem 3.1 follows using the definition of nuclear operators, Lemma 4.1 (iv) of [10] and the fact that $L^{p}$-convergence of a sequence implies the convergence of a sequence almost everywhere.

The only if part of Theorem 3.1 is a straightforward using the part (iv) of [10, Lemma 4.1] and applications of monotone convergence theorem of B. Levi and Lebesgue dominated convergence theorem.

This criterion applied to discrete and periodic operators gives the following characterizations (for the proof we refer the reader to [10]).

Theorem 3.3. Let a be a measurable function defined on $\mathbb{Z}^{n} \times \mathbb{T}^{n r}$. The multilinear pseudo-differential operator $T_{a}: L^{p_{1}}\left(\mathbb{Z}^{n}\right) \times L^{p_{2}}\left(\mathbb{Z}^{n}\right) \times \cdots L^{p_{r}}\left(\mathbb{Z}^{n}\right) \rightarrow L^{p}\left(\mathbb{Z}^{n}\right), 1 \leq p_{i}<\infty$,

[Revista Integración, temas de matemáticas 
for all $1 \leq i \leq r$, is a s-nuclear, $0<s \leq 1$, operator if, and only if, the following decomposition holds:

$$
a(x, \xi)=e^{-i 2 \pi \tilde{x} \cdot \xi} \sum_{k} h_{k}(x) \mathscr{F}_{\mathbb{Z}^{n r}}\left(g_{k}\right)(-\xi), \xi \in \mathbb{T}^{n r}, x \in \mathbb{Z}^{n},
$$

where $\tilde{x}=(x, x, \ldots, x) \in\left(\mathbb{Z}^{n}\right)^{r} ;\left\{h_{k}\right\}_{k}$ and $\left\{g_{k}\right\}_{k}$ with $g_{k}=\left(g_{k 1}, g_{k 2}, \ldots, g_{k r}\right)$ are two sequences in $L^{p}\left(\mathbb{Z}^{n}\right)$ and $L^{p_{1}^{\prime}}\left(\mathbb{Z}^{n}\right) \times L^{p_{2}^{\prime}}\left(\mathbb{Z}^{n}\right) \times \cdots \times L^{p_{r}^{\prime}}\left(\mathbb{Z}^{n}\right)$, respectively, such that $\sum_{n=1}^{\infty}\left\|h_{n}\right\|_{L^{p}\left(\mathbb{Z}^{n}\right)}^{s}\left\|g_{n}\right\|_{L^{p_{1}^{\prime}}\left(\mathbb{Z}^{n}\right) \times L^{p_{2}^{\prime}}\left(\mathbb{Z}^{n}\right) \times \cdots \times L^{p_{r}^{\prime}}\left(\mathbb{Z}^{n}\right)}^{s}<\infty$.

Similarly, we can classify the $s$-nuclearity of periodic multilinear operators.

Theorem 3.4. Let $m$ be a measurable function on $\mathbb{T}^{n} \times \mathbb{Z}^{n r}$. Then the mutlilinear pseudodifferential operator $T_{m}: L^{p_{1}}\left(\mathbb{T}^{n}\right) \times \cdots \times L^{p_{r}}\left(\mathbb{T}^{n}\right) \rightarrow L^{p}\left(\mathbb{T}^{n}\right), 1 \leq p_{i}, p<\infty$ for $1 \leq i \leq r$, is a $s$-nuclear, $0<s \leq 1$, operator if, and only if, there exist two sequences $\left\{g_{k}\right\}_{k}$ with $g_{k}=\left(g_{k 1}, g_{k 2}, \ldots, g_{k r}\right)$ and $\left\{h_{k}\right\}_{k}$ in $L^{p_{1}^{\prime}}\left(\mathbb{T}^{n}\right) \times \cdots \times L^{p_{r}^{\prime}}\left(\mathbb{T}^{n}\right), \frac{1}{p_{i}}+\frac{1}{p_{i}^{\prime}}=1$ for $1 \leq i \leq r$

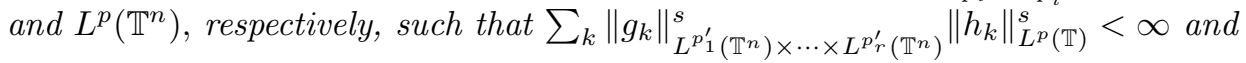

$$
m(x, \eta)=e^{-i 2 \pi \tilde{x} \cdot \eta} \sum_{k} h_{k}(x)\left(\mathscr{F}_{\mathbb{T} n r} g_{k}\right)(-\eta), \eta \in \mathbb{Z}^{n r}
$$

where $\tilde{x}=(x, x, \ldots, x) \in \mathbb{T}^{n r}$.

Now, we present the following sharp result on the $s$-nuclearity of periodic Fourier integral operators.

Theorem 3.5. Let us consider the real-valued function $\phi: \mathbb{T}^{n} \times \mathbb{Z}^{n r} \rightarrow \mathbb{R}$. Let us consider the Fourier integral operator

$$
A f(x):=\sum_{\xi \in \mathbb{Z}^{n r}} e^{i \phi\left(x, \xi_{1}, \xi_{2}, \cdots, \xi_{r}\right)} a\left(x, \xi_{1}, \xi_{2}, \cdots, \xi_{r}\right)\left(\mathscr{F}_{\mathbb{T}^{n}} f_{1}\right)\left(\xi_{1}\right) \cdots\left(\mathscr{F}_{\mathbb{T}^{n}} f_{r}\right)\left(\xi_{r}\right),
$$

with symbol satisfying the summability condition

$$
\sum_{\xi \in \mathbb{Z}^{n r}}\left\|a\left(\cdot, \xi_{1}, \xi_{2}, \cdots, \xi_{r}\right)\right\|_{L^{p}\left(\mathbb{T}^{n}\right)}^{s}<\infty .
$$

Then $A$ extends to a s-nuclear, $0<s \leq 1$, operator from $L^{p_{1}}\left(\mathbb{T}^{n}\right) \times \cdots \times L^{p_{r}}\left(\mathbb{T}^{n}\right)$ into $L^{p}\left(\mathbb{T}^{n}\right)$, provided that $1 \leq p_{j}<\infty$, and $1 \leq p \leq \infty$.

Remark 3.6. The proof of Theorem 3.5 follows using Theorem 3.1 by considering the function

$$
h_{\xi}(x):=e^{i \phi\left(x, \xi_{1}, \xi_{2}, \cdots, \xi_{r}\right)} a\left(x, \xi_{1}, \xi_{2}, \cdots, \xi_{r}\right),
$$

the functional

$$
\left\langle e_{\xi}^{\prime}, f\right\rangle:=\left(\mathscr{F}_{\mathbb{T}^{n}} f_{1}\right)\left(\xi_{1}\right) \cdots\left(\mathscr{F}_{\mathbb{T}^{n}} f_{r}\right)\left(\xi_{r}\right)
$$

and their estimates

$$
\left\|h_{k}\right\|_{L^{p}\left(\mathbb{T}^{n}\right)}=\left\|a\left(x, \xi_{1}, \xi_{2}, \cdots, \xi_{r}\right)\right\|_{L^{p}\left(\mathbb{T}_{x}^{n}\right)}
$$

and $\left|\left\langle e_{\xi}^{\prime}, f\right\rangle\right| \leq \prod_{j=1}^{r}\left\|f_{j}\right\|_{L^{p_{j}^{\prime}}}$.

Vol. 36, $\left.\mathrm{N}^{\circ} 2,2018\right]$ 
Example 3.7. In order to illustrate the previous conditions, we consider the multilinear Bessel potential. This can be introduced as follows. Consider the periodic multilinear Laplacian denoted by

$$
\mathscr{L}:=(\mathcal{L}, \cdots, \mathcal{L})
$$

acting on $f=\left(f_{1}, \cdots, f_{r}\right) \in \mathscr{D}\left(\mathbb{T}^{n}\right)^{r}$ by

$$
\begin{aligned}
\mathscr{L} f(x): & =\left(\mathcal{L} f_{1}(x)\right) \cdots\left(\mathcal{L} f_{r}(x)\right) \\
& =\sum_{\left(\xi_{1}, \cdots, \xi_{r}\right)} e^{i 2 \pi x\left(\xi_{1}+\cdots+\xi_{r}\right)}\left|\xi_{1}\right|^{2} \cdots\left|\xi_{r}\right|^{2}\left(\mathscr{F}_{\mathbb{T}^{n}} f_{1}\right)\left(\xi_{1}\right) \cdots\left(\mathscr{F}_{\mathbb{T}^{n}} f_{r}\right)\left(\xi_{r}\right) .
\end{aligned}
$$

For $r=1$, we recover the usual periodic Laplacian

$$
\mathcal{L} f(x)=-\frac{1}{4 \pi^{2}}\left(\sum_{j=1}^{n} \partial_{\theta_{j}}^{2}\right) f(x)=\sum_{\xi \in \mathbb{Z}^{n}} e^{i 2 \pi x \cdot \xi}|\xi|^{2}\left(\mathscr{F}_{\mathbb{T}^{n}} f\right)(\xi) .
$$

The multilinear Bessel potential of order $\alpha=\left(\alpha_{1}, \cdots, \alpha_{r}\right) \in \mathbb{N}_{0}^{r}$,

$$
(I+\mathscr{L})^{-\frac{\alpha}{2}}:=\left((I+\mathcal{L})^{-\frac{\alpha_{1}}{2}}, \cdots,(1+\mathcal{L})^{-\frac{\alpha_{r}}{2}}\right),
$$

can be defined by the Fourier analysis associated to the torus as

$$
\begin{aligned}
& (I+\mathscr{L})^{-\frac{\alpha}{2}} f(x)=(I+\mathcal{L})^{-\frac{\alpha_{1}}{2}} f_{1}(x) \cdots(1+\mathcal{L})^{-\frac{\alpha_{r}}{2}} f_{r}(x) \\
& =\sum_{\left(\xi_{1}, \cdots, \xi_{r}\right)} e^{i 2 \pi x\left(\xi_{1}+\cdots+\xi_{r}\right)}\left(\prod_{j=1}^{r}\left(1+\left|\xi_{j}\right|^{2}\right)^{-\frac{\alpha_{j}}{2}}\right)\left(\mathscr{F}_{\mathbb{T}^{n}} f_{1}\right)\left(\xi_{1}\right) \cdots\left(\mathscr{F}_{\mathbb{T}^{n}} f_{r}\right)\left(\xi_{r}\right) .
\end{aligned}
$$

From the estimate

$$
a(x, \xi)=\prod_{j=1}^{r}\left(1+\left|\xi_{j}\right|^{2}\right)^{-\frac{\alpha_{j}}{2}} \leq \prod_{j=1}^{r}\left(1+\left|\xi_{j}\right|^{2}\right)^{-\min _{1 \leq j \leq r}\left\{\frac{\alpha_{j}}{2}\right\}} \lesssim\langle\xi\rangle^{-\min _{1 \leq j \leq r}\left\{\alpha_{j}\right\}},
$$

Theorem 3.5 applied to $a(x, \xi)=\prod_{j=1}^{r}\left(1+\left|\xi_{j}\right|^{2}\right)^{-\frac{\alpha_{j}}{2}}$ implies that the multilinear Bessel potential $(I+\mathscr{L})^{-\frac{\alpha}{2}}$ extends to a s-nuclear operator from $L^{p_{1}}\left(\mathbb{T}^{n}\right) \times \cdots \times L^{p_{r}}\left(\mathbb{T}^{n}\right)$ into $L^{p}\left(\mathbb{T}^{n}\right)$ for all $1 \leq p_{j}<\infty$ and $1 \leq p \leq \infty$, provided that

$$
\varkappa:=\min _{1 \leq j \leq r}\left\{\alpha_{j}\right\}>n r / s .
$$

This conclusion is sharp, in the sense that if we restrict our analysis to $r=1$ and $p_{1}=p=2$, the operator $(I+\mathcal{L})^{-\frac{\alpha}{2}}$ extends to a s-nuclear operator on $L^{2}\left(\mathbb{T}^{n}\right)$ if, and only if, $\varkappa:=\alpha>n r / s=n / s$.

Example 3.8. Now, we consider FIOs with symbols admitting some type of singularity at the origin. In this general context, let us choose a sequence $\kappa \in L^{s}\left(\mathbb{Z}^{n r}\right)$. Let us consider the symbol

$$
a(x, \xi):=\frac{1}{|x|^{\rho}} \kappa(\xi), x \in \mathbb{T}^{n}, x \neq 0, \xi \in \mathbb{Z}^{n r}, \rho>0 .
$$

[Revista Integración, temas de matemáticas 
If we consider the Fourier integral operator associate to $a(\cdot, \cdot)$,

$$
A f(x):=\sum_{\xi \in \mathbb{Z}^{n r}} e^{i \phi\left(x, \xi_{1}, \xi_{2}, \cdots, \xi_{r}\right)} \frac{1}{|x|^{\rho}} \kappa\left(\xi_{1}, \cdots, \xi_{r}\right)\left(\mathscr{F}_{\mathbb{T}^{n}} f_{1}\right)\left(\xi_{1}\right) \cdots\left(\mathscr{F}_{\mathbb{T}^{n}} f_{r}\right)\left(\xi_{r}\right),
$$

the condition

$$
0<\rho<n / p
$$

implies that the periodic Fourier integral operator $A$ extends to a s-nuclear multilinear operator from $L^{p_{1}}\left(\mathbb{T}^{n}\right) \times \cdots \times L^{p_{r}}\left(\mathbb{T}^{n}\right)$ into $L^{p}\left(\mathbb{T}^{n}\right)$, for all $1 \leq p_{j}<\infty$ and $1 \leq p \leq \infty$. In fact, by Theorem 3.5, we only need to verify that

$$
\sum_{\xi \in \mathbb{Z}^{n r}}\left\|a\left(\cdot, \xi_{1}, \xi_{2}, \cdots, \xi_{r}\right)\right\|_{L^{p}\left(\mathbb{T}^{n}\right)}^{s}=\left(\int_{\mathbb{T}^{n}} \frac{d x}{|x|^{p^{\cdot} \cdot}}\right)^{\frac{s}{p}} \sum_{\xi \in \mathbb{Z}^{n r}}|\kappa(\xi)|^{s}<\infty .
$$

But, for every $\rho>0$, this happens if, and only if, $0<\rho<n / p$.

\section{Acknowledgment}

Vishvesh Kumar thanks the Council of Scientific and Industrial Research, India, for its senior research fellowship. Duván Cardona was partially supported by Pontificia Universidad Javeriana, Mathematics Department. We would like to thank the anonymous referee for his/her careful evaluation which helped to improve the presentation of this work.

\section{References}

[1] Agranovich M.S., "Spectral properties of elliptic pseudo-differential operators on a closed curve", Funct. Anal. Appl. 13 (1971), 279-281.

[2] Aoki S., "On the boundedness and the nuclearity of pseudo-differential operators", Comm. Partial Diff. Equations 6 (1981), No. 8, 849-881.

[3] Bényi A., Bernicot F., Maldonado D., Naibo V. and Torres R., "On the Hörmander classes of bilinear pseudo-differential operators II", Indiana Univ. Math. J. 62 (2013), 1733-1764.

[4] Bényi A., Maldonado D., Naibo V. and Torres R., "On the Hörmander classes of bilinear pseudodifferential operators", Integral Equ. Oper. Theory 67 (2010), 341-364.

[5] Botchway L., Kibiti G. and Ruzhansky M., "Difference equations and pseudo-differential

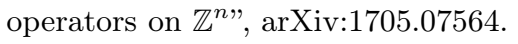

[6] Cardona D. "Estimativos $L^{2}$ para una clase de operadores pseudodiferenciales definidos en el toro", Rev. Integr. Temas Mat. 31 (2013), No. 2, 142-157.

[7] Cardona D., "Weak type $(1,1)$ bounds for a class of periodic pseudo-differential operators", J. Pseudo-Differ. Oper. Appl. 5 (2014), No. 4, 507-515.

[8] Cardona D., "On the boundedness of periodic pseudo-differential operators", Monatsh. Math. 185 (2017), No. 2, 189-206.

Vol. 36, $\left.\mathrm{N}^{\circ} 2,2018\right]$ 
[9] Cardona D., "Pseudo-differential operators on $\mathbb{Z}^{n}$ with applications to discrete fractional integral operators", arXiv:1803.00231.

[10] Cardona D. and Kumar V., " $L^{p}$-boundedness and $L^{p}$-nuclearity of multilinear pseudodifferential operators on $Z^{n}$ and the torus $T^{n "}$, arXiv:1809.08380.

[11] Cardona D., Messiouene R. and Senoussaoui A., " $L^{p}$-bounds for periodic Fourier integral operators (Fourier series operators)", arXiv:1807.09892.

[12] Catana V., " $L^{p}$-boundedness of Multilinear Pseudo-differential Operators on $\mathbb{Z}^{n}$ and $\mathbb{T}^{n}$ ", Math. Model. Nat. Phenom. 9 (2014), No. 5, 17-38.

[13] Coifman R. and Meyer Y., "On commutators of singular integrals and bilinear singular integrals", Trans. Amer. Math. Soc. 212 (1975), 315-331.

[14] Coifman R. and Meyer Y., Ondelettes et operateurs III. Operateurs multilineaires, Hermann, Paris, (1991).

[15] Delgado J., " $L^{p}$ bounds for pseudo-differential operators on the torus", Oper. Theory Adv. Appl. 231 (2012), 103-116.

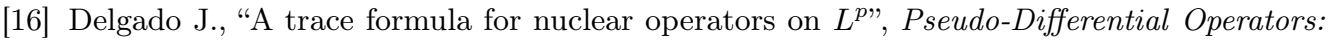
Complex Analysis and Partial Differential Equations, Operator Theory: Advances and Applications, in: Schulze, B.W., Wong, M.W. (eds.) 205, Birkhäuser, Basel (2010), 181-193.

[17] Delgado J. and Wong M.W., " $L^{p}$-nuclear pseudo-differential operators on $\mathbb{Z}$ and $\mathbb{S}^{1}$ ", Proc. Amer. Math. Soc. 141 (2013), No. 11, 3935-3944.

[18] Delgado J., "The trace of nuclear operators on $L^{p}(\mu)$ for $\sigma$-finite Borel measures on second countable spaces", Integr. Equ. Oper. Theory 68 (2010), No. 1, 61-74.

[19] Fujita M. and Tomita N., "Weighted norm inequalities for multilinear Fourier multipliers", Trans. Amer. Math. Soc. 364 (2012), No. 12, 6335-6353.

[20] Ghaemi M. B., Jamalpour Birgani M. and Wong M.W., "Characterizations of nuclear pseudo-differential operators on $\mathbb{S}^{1}$ with applications to adjoints and products", J. PseudoDiffer. Oper. Appl. 8 (2017), No. 2, 191-201.

[21] Grafakos L., Miyachi A. and Tomita N., "On multilinear Fourier multipliers of limited smoothness", Canad. J. Math. 65 (2013), 299-330.

[22] Grafakos L. and Si Z., "The Hörmander multiplier theorem for multilinear operators", J. Reine Angew. Math. 668 (2012), 133-147.

[23] Grafakos L. and Torres R., "Discrete decompositions for bilinear operators and almost diagonal conditions", Trans. Amer. Math. Soc. 354 (2012), 1153-1176.

[24] Grafakos L. and Torres R., "Multilinear Calderón-Zygmund theory", Adv. Math. 165 (2002), 124-164.

[25] Hörmander L., The Analysis of the linear partial differential operators Vol. III, IV SpringerVerlag, (1985).

[26] Jamalpour Birgani M., "Characterizations of Nuclear Pseudo-differential Operators on $\mathbb{Z}$ with some Applications", Math. Model. Nat. Phenom. 13 (2018), 13-30.

[Revista Integración, temas de matemáticas 
[27] Kenig C. and Stein E., "Multilinear estimates and fractional integration", Math. Res. Lett. 6 (1999), 1-15.

[28] Kumar V., "Pseudo-differential operators on homegeneous spaces of Compact and Hausdorff groups", to appear in Forum Mathematicum, (2018).

[29] Mclean W.M., "Local and Global description of periodic pseudo-differential operators", Math. Nachr. 150 (191), 151-161.

[30] Michalowski N., Rule D. and Staubach W., "Multilinear pseudodifferential operators beyond Calderón-Zygmund operators", J. Math. Anal. Appl. 414 (2014), 149-165.

[31] Miyachi A. and Tomita N., "Minimal smoothness conditions for bilinear Fourier multipliers", Rev. Mat. Iberoam. 29 (2013), 495-530.

[32] Miyachi A. and Tomita N. "Calderón-Vaillancourt type theorem for bilinear operators", Indiana Univ. Math. J. 62 (2013), 1165-1201.

[33] Miyachi A. and Tomita N. "Bilinear pseudo-differential operators with exotic symbols", to appear in Ann. Inst. Fourier (Grenoble), arXiv:180106744.

[34] Molahajloo S., "A characterization of compact pseudo-differential operators on $\mathbb{S}^{1}$ ", Oper. Theory Adv. Appl. 213 (2011), 25-29.

[35] Molahajloo S. and Wong M.W., "Pseudo-differential Operators on $\mathbb{S}^{1}$ ", New developments in pseudo-differential operators, Eds. L. Rodino and M.W. Wong. 297-306 (2008).

[36] Molahajloo S. and Wong M.W., "Ellipticity, Fredholmness and spectral invariance of pseudo-differential operators on $\mathbb{S}^{1 "}$ J. Pseudo-Differ. Oper. Appl. 1 (2010), No. 2, 183-205.

[37] Muscalu C., Tao T. and Thiele C., "Multilinear operators given by singular multipliers", J. Amer. Math. Soc. 15 (2002), 469-496.

[38] Rabinovich V.S., "Exponential estimates of solutions of pseudo-differential equations on the lattice $(\mu \mathbb{Z})^{n}$, applications to the lattice Schrödinger and Dirac operators", J. Pseudo-Differ. Oper. Appl. 1 (2010), No. 2, 233-253.

[39] Rabinovich V.S., "Wiener algebra of operators on the lattice $(\mu \mathbb{Z})^{n}$ depending on the small parameter $\mu>0$ ", Complex Var. Elliptic Equ. 58 (2013), No. 6, 751-766.

[40] Rabinovich V.S. and Roch S., "The essential spectrum of Schrödinger operators on lattices", J. Phys. A. 39 (2006), No. 26, 8377-8394.

[41] Rabinovich V.S. and Roch S., "Essential spectra and exponential estimates of eigenfunctions of lattice operators of quantum mechanics", J. Phys. A. 42 (2009), No. 38, 385207.

[42] Rempala J.A., "On a proof of the boundedness and nuclearity of pseudodifferential operators in $\mathbb{R}^{n ",}$ Annales Polonici Mathematici 52 (1990), 59-65.

[43] Rodriguez C.A., " $L^{p}$-estimates for pseudo-differential operators on $\mathbb{Z}^{n}$ ", J. Pseudo-Differ. Oper. Appl. 2 (2011), 367-365.

[44] Ruzhansky M. and Turunen V., "Quantization of Pseudo-Differential Operators on the Torus", J. Fourier Anal. Appl. 16 (2010), 943-982.

[45] Ruzhansky M. and Turunen V., Pseudo-differential Operators and Symmetries: Background Analysis and Advanced Topics, Birkhaüser-Verlag, Basel, (2010).

Vol. 36, $\left.\mathrm{N}^{\circ} 2,2018\right]$ 
[46] Tomita N., "A Hörmander type multiplier theorem for multilinear operators", J. Funct. Anal. 259 (2010), 2028-2044.

[47] Turunen V. and Vainikko G., "On symbol analysis of periodic pseudodifferential operators, Z. Anal. Anwendungen 17 (1998), 9-22.

[48] Stein E. and Weiss G., Introduction to Fourier analysis on Euclidean spaces, Princeton University Press, Princeton, N.J. (1971). 